\title{
Orthogeriatric services associated with lower 30-day mortality for older patients who undergo surgery for hip fracture
}

\section{Justin Zeltzer \\ nometrics), LLB \\ Biostatistician \\ Rebecca J Mitchell \\ MA(Psych), MOHS, PhD \\ Senior Research Fellow ${ }^{1,2}$ \\ Barbara Toson \\ BStatEc \\ Statistician \\ Ian A Harris \\ MBBS, MMed(ClinEpi), PhD \\ Professor of Orthopaedic \\ Surgery ${ }^{3}$ \\ Laura Ahmad \\ MD, PhD, FRACP \\ Chair, Orthogeriatrics Group \\ and Geriatrician \\ Jacqui Close MD, FRCP, FRAC \\ Director \\ 1 Falls and Injury Prevention Group, Neuroscience Research Australia, Sydney, \\ 2 Transport and Road Safety Research, University of \\ New South Wales, \\ Sydney, NSW. \\ 3 South Western Sydney \\ Clinical School, \\ University of \\ New South Wales, \\ Sydney, NSW. \\ 4 Aged Health Network \\ Agency for Clinical \\ Innovation, \\ Sydney, NSW \\ 5 Department of Aged \\ Care, North Shore Private \\ Hospital, Sydney, NSW. \\ r.mitchell@unsw.edu.au}

MJA 2014; 201: 409-411 doi: 10.5694/mjal4.00055

Online first 29/09/14 ip fracture is one of the most common, serious and costly

injuries sustained by older people; it often results in disability and a need for enhanced levels of care, and it occasionally results in death. ${ }^{1-3}$

To improve outcomes after hip fracture, various models of care have been proposed and evaluated. ${ }^{1,4,5}$ One study has shown reductions in morbidity and mortality, ${ }^{1}$ another has produced inconclusive results, ${ }^{6}$ and a systematic review has found that hip fracture patients who receive multidisciplinary inpatient rehabilitation tend to achieve better outcomes, although it could not show this to a significant level.?

Orthogeriatric care is medical care for older patients with orthopaedic conditions that is provided collaboratively with the treating orthopaedic team. In Australia, it is predominantly provided by geriatricians. We examined the impact of orthogeriatric services on 30-day mortality and length of stay (LOS) for hip fracture patients undergoing surgery in public hospitals in New South Wales.

\section{Methods}

We conducted a retrospective analysis of patients aged 65 years and older who had a fractured hip and received surgical intervention between 1 July 2009 and 30 June 2011 at one of the 37 NSW public hospitals operating on hip fracture patients. Ethics approval was obtained from the NSW Population and Health Services Research Ethics Committee.

\section{Data sources}

Patient data were obtained from the NSW Admitted Patient Data Collection (APDC), which contains information on inpatient separations from all hospitals in NSW. It holds information on patient demographics, diagnoses, separation types and clinical procedures. For each patient,

\section{Abstract}

Objective: To examine the impact of orthogeriatric services on 30-day mortality and length of stay (LOS) for hip fracture patients undergoing surgery in public hospitals in New South Wales.

Design, setting and patients: A retrospective analysis of patients aged 65 years and older who had a fractured hip and received surgical intervention between 1 July 2009 and 30 June 2011 at one of the 37 NSW public hospitals operating on hip fracture patients.

Main outcome measures: 30-day mortality and LOS.

Results: During the study period, there were 9601 hip fracture cases for which surgery was done. Mean age, sex and comorbidity distribution were similar for hip fracture patients treated in hospitals with an orthogeriatric service compared with those treated in hospitals without an orthogeriatric service. There were 706 deaths within 30 days of hip fracture surgery, and the overall unadjusted 30-day mortality rate was $7.4 \%$. The median adjusted 30 -day mortality rate for hospitals with an orthogeriatric service was significantly lower than that for hospitals without an orthogeriatric service (6.2\% v 8.4\%; $P<0.002$ ). Median total LOS was longer at hospitals with an orthogeriatric service compared with hospitals that did not have an orthogeriatric service ( 26 days $\vee 22$ days; $P<0.001$ ).

Conclusions: The presence of an orthogeriatric service was associated with a reduction in 30-day mortality but a longer LOS. More research is required to understand the key aspects of care that determine health outcomes. The recently launched Australian and New Zealand Hip Fracture Registry will provide data that will enable improvements in care.

individual episodes of care were linked to form one record. The diagnoses and external cause codes are classified using the International Classification of Diseases, 10th revision, Australian modification (ICD-10-AM). ${ }^{8}$ Mortality data were obtained from the NSW Registry of Births, Deaths and Marriages (RBDM).

\section{Identification of cases}

Hip fracture patients undergoing surgical intervention were identified as those for whom the principal diagnosis was ICD-10-AM code S72.0, S72.1 or S72.2 (hip fracture) and the principal procedure code was ICD-10AM code 47519-00, 47522-00, 47528-01, 47531-00, 49318-00 or 49319-00 (hip fracture interventions).

\section{Data linkage}

The APDC was linked to the RBDM mortality data by the Centre for Health Record Linkage. This enabled identification of the first admission for a hip fracture per person, any public or private hospital transfers related to the admission where a hip fracture procedure occurred on or after a hip fracture diagnosis, and any death within 30 days of surgery.

\section{Identification of comorbidities}

The Charlson comorbidity index (CCI) ICD-10 Deyo adaptation, ${ }^{9}$ with a 1-year look-back period, was used to identify comorbidities and for casemix adjustment. The number of comorbidities were summed for each patient, and patients were divided into three groups based on CCI scores $(0 ; 1$ or 2 ; and $\geqslant 3)$.

\section{Identification of hospitals with orthogeriatric services}

In 2012, a facility-level audit of all public hospitals in NSW providing surgery for hip fracture showed that $62 \%$ of hospitals provided some form of orthogeriatric care. ${ }^{10}$ Hospitals with an orthogeriatric service in 2012 were contacted to establish whether the service was available during the period 1 July 2009 to 30 June 2011. 
1 Characteristics of hip fracture cases for which surgery was performed in public hospitals with and without an orthogeriatric service, New South Wales, July 2009 - June 2011

\begin{tabular}{|c|c|c|c|}
\hline Characteristic & $\begin{array}{c}\text { Hospitals with } \\
\text { orthogeriatric service } \\
(n=14)\end{array}$ & $\begin{array}{c}\text { Hospitals without } \\
\text { orthogeriatric service } \\
(n=23)\end{array}$ & $P$ \\
\hline No. of surgeries & 4575 & 5026 & - \\
\hline Mean (SD) patient age, years & $84.4(7.4)$ & $84.2(7.5)$ & 0.20 \\
\hline No. (\%) of surgeries on men & $1239(27.1 \%)$ & $1326(26.4 \%)$ & 0.44 \\
\hline $\mathrm{CCl}$ score group & & & 0.36 \\
\hline 0 & 2715 (59.3\%) & 3018 (60.0\%) & \\
\hline 1 or 2 & $1256(27.5 \%)$ & $1393(27.7 \%)$ & \\
\hline$\geqslant 3$ & 604 (13.2\%) & $615(12.2 \%)$ & \\
\hline Overall 30-day patient mortality rate & $6.5 \%$ & $8.1 \%$ & 0.002 \\
\hline $\begin{array}{l}\text { Median (IQR) 30-day hospital mortality rate - } \\
\text { unadjusted }\end{array}$ & $6.6 \%(2.1 \%)$ & $8.2 \%(2.3 \%)$ & $<0.02$ \\
\hline $\begin{array}{l}\text { Median (IQR) 30-day hospital mortality rate - } \\
\text { adjusted* }\end{array}$ & $6.2 \%(2.1 \%)$ & $8.4 \%(2.4 \%)$ & $<0.002$ \\
\hline \multicolumn{4}{|l|}{ Acute length of stay, days ${ }^{\dagger}$} \\
\hline Mean (SD) & $12.9(10.9)$ & $11.2(9.1)$ & $<0.001$ \\
\hline Median (IQR) & $10(8)$ & $9(8)$ & $<0.001$ \\
\hline \multicolumn{4}{|l|}{ Total length of stay, days ${ }^{\dagger}$} \\
\hline Mean (SD) & $30.1(22.8)$ & $28.7(29.5)$ & $<0.01$ \\
\hline Median (IQR) & $26(31)$ & $22(29)$ & $<0.001$ \\
\hline \multicolumn{4}{|l|}{ Time from admission to surgery, days } \\
\hline Mean (SD) & $1.8(2.7)$ & $1.7(13.2)$ & 0.63 \\
\hline Median (IQR) & $1(2)$ & $1(2)$ & $<0.001$ \\
\hline
\end{tabular}

$\mathrm{CCl}=$ Charlson comorbidity index. $\mathrm{IQR}=$ interquartile range. * Adjusted for age, sex and CCl score group. $\uparrow$ Acute length of stay is the cumulative length of stay for all linked acute episodes of care (defined by the acute flag in the NSW Admitted Patient Data Collection); total length of stay also includes the linked non-acute episodes of care. 30-day mortality rate for hospitals with an orthogeriatric service was significantly lower than that for hospitals without an orthogeriatric service $(6.2 \%$ v $8.4 \% ; P<0.002)$ (Box 1 ). The median 30-day adjusted mortality rate for hospitals that are major trauma centres was not significantly different to that for hospitals that are not major trauma centres $(7.2 \%$ and $7.8 \%$, respectively; $P=0.3$ ).

\section{Discussion}

In our study, hospitals with an orthogeriatric service had lower 30-day mortality rates and longer LOS for patients who underwent surgery for hip fracture.

The difference in mortality rates may be due to medical optimisation before surgery, prevention and early detection of medical complications, better coordination of care, better communication between staff responsible for care, and better management of comorbidities. Results of other studies indicate that respiratory disease and cardiac disease are the biggest causes of mortality after hip fracture and that these diseases are likely to be managed better in hospitals with an orthogeriatric service. ${ }^{1,6,11}$ Equally, it could be argued that the presence of an orthogeriatric service reflects a better resourced hospital that provides treatment for larger numbers of hip fracture patients.

Various reasons can be put forward to explain the differences in LOS. Given that $50 \%$ of deaths occurred within 12.5 days of admission to hospital, it may simply be the survival effect that produced longer LOS in hospitals with an orthogeriatric service, although this is unlikely to solely explain the difference that we found. Without looking at readmission figures, it is difficult to know whether the longer LOS is appropriate and whether premature discharge may lead to higher rates of readmission.

Other studies that have examined mortality and LOS after hip fracture surgery at hospitals with and without specialised orthogeriatric or multidisciplinary services over the past decade have produced mixed results.1,6,12-14 
2 Mean number of hip fracture surgeries per year and 30-day mortality in public hospitals with and without an orthogeriatric service, New South Wales,

July 2009 - June 2011*

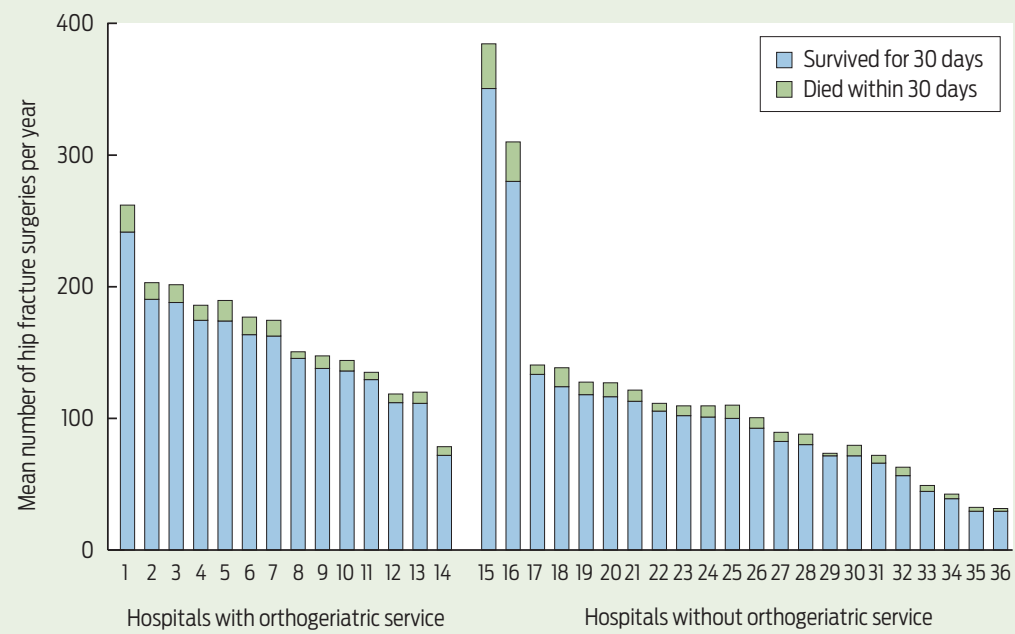

* One hospital without an orthogeriatric service was omitted from the analysis due to low number of surgeries.

3 Adjusted 30-day mortality rates after hip fracture surgery in public hospitals according to presence or absence of an orthogeriatric service and by major trauma centre status, New South Wales, July 2009 - June 2011*

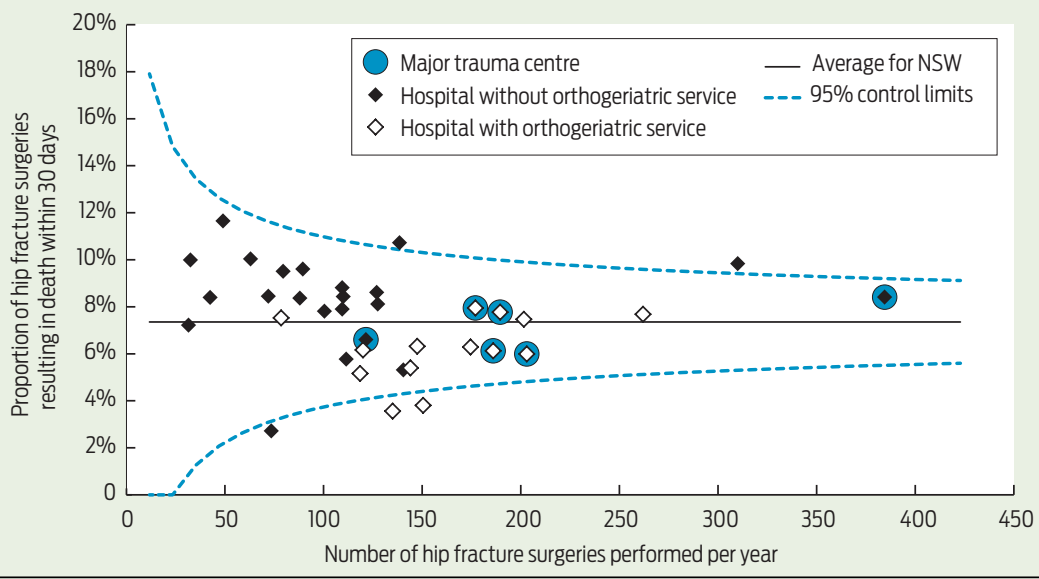

* One hospital without an orthogeriatric service was omitted from the analysis due to low number of surgeries.

Our study has four main limitations. First, the approach to delivery of orthogeriatric care varies across hospitals (eg, in some hospitals, physicians other than geriatricians provide medical input for hip fracture patients), and we did not account for such differences. Second, the APDC did not include information on cognitive state, prefracture ambulatory status or preadmission place of residence, which are predictors of health outcomes. ${ }^{6}$ While comorbidity is an important predictor of outcomes, it does not reflect disease severity. Third, we did not extract information on postoperative complications, which have been shown to differ based on the availability of an orthogeriatric service.,6,11,15 Finally, we did not explore the potential for backwards causation due to better overall standards of care in hospitals that have an orthogeriatric service.

Time from hip fracture to surgery has been shown to affect health outcomes and LOS. ${ }^{16}$ The APDC does not record exact time from fracture to surgery, so we used a proxy measure (days between admission for first recorded hip fracture diagnosis and surgery).
In the United Kingdom, the establishment of the National Hip Fracture Database has been instrumental in driving changes in clinical care and patient outcomes, along with improvements in hospital services for treatment of hip fracture patients. As a result of many changes in the UK, 30-day mortality has decreased significantly. ${ }^{17}$ Similar work is now underway in Australia: national guidelines and standards of care are being developed, and a minimum patient-level dataset (available as a patient audit form) has been prepared to facilitate the recently launched Australian and New Zealand Hip Fracture Registry (http://www. anzhfr.org). Data collected by the Registry will be integral to future research on the key determinants of health outcomes and will help create more effective services for hip fracture patients.

Acknowledgements: Justin Zeltzer was supported by the NSW Ministry of Health's NSW Biostatistical Officer Training Program. The Falls and Injury Prevention Group is partly supported by the NSW Ministry of Health. We thank the Centre for Health Record Linkage for conducting the record linkage and the Centre for Epidemiology and Evidence at the NSW Ministry of Health for providing access to Secure Analytics for Population Health Research and Intelligence (a data warehouse and analysis tool that enables health dataset analysis)

Competing interests: No relevant disclosures.

Received 14 Jan 2014, accepted 12 Jun 2014

1 Fisher AA, Davis MW, Rubenach SE, et al. Outcomes for older patients with hip fractures: the impact of orthopedic and geriatric medicine cocare. J Orthop Trauma 2006; 20: 172-180.

2 Brown P, McNeill R, Leung W, et al. Current and future economic burden of osteoporosis in New Zealand. Appl Health Econ Health Policy 2011; 9: 111-123.

3 Randell A, Sambrook PN, Nguyen TV, et al. Direct clinical and welfare costs of osteoporotic fractures in elderly men and women. Osteoporos Int 1995; 5 : 427-432.

4 Chong CP, Savige J, Lim WK. Orthopaedic-geriatric models of care and their effectiveness. Australas J Ageing 2009; 28: 171-176.

5 Kammerlander C, Roth T, Friedman SM, et al. Ortho-geriatric service - a literature review comparing different models. Osteoporos Int 2010; 21 Suppl 4: S637-S646.

6 Vidán M, Serra JA, Moreno C, et al. Efficacy of a comprehensive geriatric intervention in older patients hospitalized for hip fracture: a randomized, controlled trial. J Am Geriatr Soc 2005; 53: 1476-1482.

7 Handoll HH, Cameron ID, Mak JC, Finnegan TP. Multidisciplinary rehabilitation for older people with hip fractures. Cochrane Database Syst Rev 2009; (4): CD007125.

8 National Centre for Classification in Health. The International Statistical Classification of Diseases and Related Health Problems, Tenth Revision, Australian Modification (ICD-10-AM). 5th ed. Sydney: NCCH, 2006.

9 Sundararajan V, Henderson T, Perry C, et al. New ICD-10 version of the Charlson Comorbility Index predicted in-hospital mortality. J Clin Epidemiol 2004; 57: 1288-1294.

10 Australian and New Zealand Hip Fracture Registry. Facility level audit of hospitals in NSW performing surgery for hip fracture. June 2013. http://www. anzhfr.org/2012-06-20-13-08-40/australian-news/8-news/47-2013-facilitylevel-audit (accessed Sep 2014).

11 Adunsky A, Arad M, Levi R, et al. Five-year experience with the 'Sheba' model of comprehensive orthogeriatric care for elderly hip fracture patients. Disabil Rehabil 2005; 27: 1123-1127.

12 Gilchrist WJ, Newman RJ, Hamblen DL, Williams BO. Prospective randomised study of an orthopaedic geriatric patient service. BMJ 1988; 297: 1116-1118.

13 Naglie G, Tansey C, Kirkland JL, et al. Interdisciplinary inpatient care for elderly people with hip fracture: a randomized controlled trial. CMAJ 2002; 167: 25-32.

14 Swanson CE, Day GA, Yelland CE, et al. The management of elderly patients with femoral fractures. A randomised controlled trial of early intervention versus standard care. Med J Aust 1998; 169: 515-518.

15 Sivakumar BS, McDermott LM, Bell JJ, et al. Dedicated hip fracture service: implementing a novel model of care. ANZ J Surg 2013; 83: 559-563.

16 Khan SK, Kalra S, Khanna A, et al. Timing of surgery for hip fractures: a systematic review of 52 published studies involving 291,413 patients. Injury 2009; 40: 692-697.

17 British Geriatrics Society. The National Hip Fracture Database national report 2012. London: BGS, 2012. 